$\mathbb{T}$ periodica polytechnica

Mechanical Engineering

$53 / 1(2009) 49 \sqrt{53}$

doi: 10.3311/pp.me.2009-1.07

web: http://www.pp.bme.hu/me

(c) Periodica Polytechnica 2009

RESEARCH ARTICLE

\section{A method of optical roughness measurement on ultrafine metal surfaces}

\author{
Imre Péczeli / István Várkonyi
}

Received 2010-01-18

\begin{abstract}
In the article, an optical method is described to measure the surface roughness on ultrafine machined metal surfaces. The method is capable for measurement of surface roughness without mechanical contact, and without removing the work piece from the machine.

The traditional stylus-type devices require direct physical contact, which leaves scratches on the parts, whereas this optical method leaves the parts untouched. It is important to mention in advance that the characteristic magnitude of the roughness has to be below the wavelength of the applied electromagnetic wave, since this assumption is taken strictly in consideration of our method. If we apply any diode laser which operates in the typical wavelength range of 0.6-1 $\mu \mathrm{m}$, in such a case the adequate average roughness is below $0.1 \mu \mathrm{m}$, we may speak in this sense about ultrafine machining.
\end{abstract}

\section{Keywords \\ ultrafine machining $\cdot$ surface $\cdot$ roughness $\cdot$ electromagnetic wave diffraction}

Imre Péczeli

Department of Atomic Physics, BME, H-1521 Budapest,, Hungary

István Várkonyi

Consulting Kft, H-1117 Budapest, Baranyai u. 17., Hungary

\section{Introduction}

The optical measurement of topography of machined metal surfaces has been approached different directions in the past [1][3]. Bennett and Pourteus were who extended the profilometry to the noncontact direction [4]. They gave in their paper a complete expression at normal incidence to the reflectance. In this work they used Davies' [5] results which were developed in connection with the scattering of radar waves on sea surface. In this theory the water surface was characterized by Gaussian height distribution. A fundamental difference between the machined metal surface and the water surface is evident. Namely the sea surface is a dynamic, whereas the metal surface is a static system. Stover started on another way, he developed a new conception in this field. Stover set out from that fact, that any arbitrary surface profile can be thought of as being composed of a large number of sinusoidal surfaces of various amplitudes and spatial frequencies, directing in various directions in a plane. The actual surface was considered as the superposition of the above sinusoidal surfaces [6]. Our work points out that using Kirchhoff scalar diffraction theorem in far-field approximation the total diffracted and the specular reflected powers determine the average roughness $(\mathrm{Ra})$, if the wavelength is far much as the magnitude of roughness.

\section{Theory}

This theory aims to measure plain surfaces. The machined surface is illuminated by a monochromatic light beam and the propagation properties of the developed amplitude on the investigated surface are determined by the surface roughness. With respect to the ultrafine investigations, we take the following assumptions:

1 The deviation of the surface from an imaginary plane is described by a scalar-vector function $S=z-\xi(x, y)$ which at $S=0$ means the $z=\xi(x, y)$ surface. $\xi(x, y)$ is the deviation in every $(\mathrm{x}, \mathrm{y})$ point from the plane that is characterized by $z=0$. The absolute value of $\xi(x, y)$ is much smaller than the applied wavelength $|\xi(x, y)|\langle\langle\lambda$.

2 The illuminating beam is plane wave. 
3 The machined metal surface is perfect in reflection $(R=1)$.

4 The reflected amplitude on the surface is determined by the local surface geometry and by the incident beam.

5 The beam propagation is determined by the Kirchhoff scalar theory and the observation happens in the far-field.

Let us imagine a fine machined metal surface in the $z=0$ plain, as it was said this surface is given by the function $z=\xi(x, y)$. In every point of this surface, there is the electric field amplitude $\varphi(x, y, \xi(x, y))$ that is developed by the illumination and reflection on the metal surface. The $z=\xi(x, y)$ surface divides the whole space two half spaces. In a $\mathbf{r}_{p}=\left(x_{p}, y_{p}, z_{p}\right)$ point of the right half space we can give the field amplitude by the Kirchhoff integral formula:

$$
\begin{gathered}
\varphi\left(x_{p}, y_{p}, z_{p}\right)=\frac{1}{4 \pi} \int\left\{\varphi(x, y, \xi(x, y)) \operatorname{grad} g_{p}-g_{p}\right. \\
\operatorname{grad} \varphi(x, y, \xi(x, y))\} \mathbf{n} d S .
\end{gathered}
$$

In the integral formula $g_{p}$ has a singularity of $\frac{1}{\varepsilon}$ and its gradient $\left|\operatorname{grad} g_{p}\right|$ has a singularity of $\frac{1}{\varepsilon^{2}}$ in the $\mathbf{r}_{p}$ point, where $\varepsilon$ marks the radius of the sphere around the $\mathbf{r}_{p}$ point. The vector $\mathbf{r}=(x, y, \xi)$ indicates an arbitrary point of the illuminated surface. See the Fig. 1

In the integral $\mathbf{n}$ means the normal vector of the investigated surface element in the $\mathbf{r}$ point and the dS is the magnitude of the surface element. One of the most familiar possible $g_{p}$ function is [7]:

$$
g_{p}=\frac{e^{\left.i k\left(\mathbf{r}-\mathbf{r}_{p}\right)\right)}}{\left|\mathbf{r}-\mathbf{r}_{p}\right|}-\frac{e^{i k\left(\mathbf{r}-\mathbf{r}_{p-}\right)}}{\left|\mathbf{r}-\mathbf{r}_{p-}\right|}=\frac{e^{i k R}}{R}-\frac{e^{i k R^{\prime}}}{R^{\prime}}
$$

where $R=\left|\mathbf{r}-\mathbf{r}_{p}\right|$ and $R^{\prime}=\left|\mathbf{r}-\mathbf{r}_{p-}\right|$.

while $\mathbf{r}=\left(\begin{array}{l}x \\ y \\ \xi(x, y)\end{array}\right)$ arbitrary point of the investigated

surface

$$
\begin{aligned}
& \mathbf{r}_{p}=\left(\begin{array}{l}
x_{p} \\
y_{p} \\
z_{p}
\end{array}\right) \text { any observation point in the right half space } \\
& \mathbf{r}_{p-}=\left(\begin{array}{l}
x_{p} \\
y_{p} \\
-z_{p}
\end{array}\right) \text { mirror vector of } \mathbf{r}_{p} \text { with respect to the } \mathrm{z}=0
\end{aligned}
$$

plane.

The $k$ indicates the wave number of the illuminating wave $k=\frac{2 \pi}{\lambda}$.

Since at the ultrafine machining the $|\xi(x, y)| \ll \lambda$ so if the $\left(z_{p} \gg \lambda\right)$ observation point is in the far-field then the $g_{p}(x, y, \xi(x, y)) \cong 0$. This statement can be easily proved since in this case $R=\left|\mathbf{r}-\mathbf{r}_{p}\right|$ and $R^{\prime}=\left|\mathbf{r}-\mathbf{r}_{p-}\right|$ are high accuracy equals.

Using the expansion of $R$ and $R^{\prime}$

$$
\begin{aligned}
& R=\sqrt{\left(x-x_{p}\right)^{2}+\left(y-y_{p}\right)^{2}+\left(\xi(x, y)-z_{p}\right)^{2}} \\
& \text { and } \\
& R^{\prime}=\sqrt{\left(x-x_{p}\right)^{2}+\left(y-y_{p}\right)^{2}+\left(\xi(x, y)+z_{p}\right)^{2}}
\end{aligned}
$$

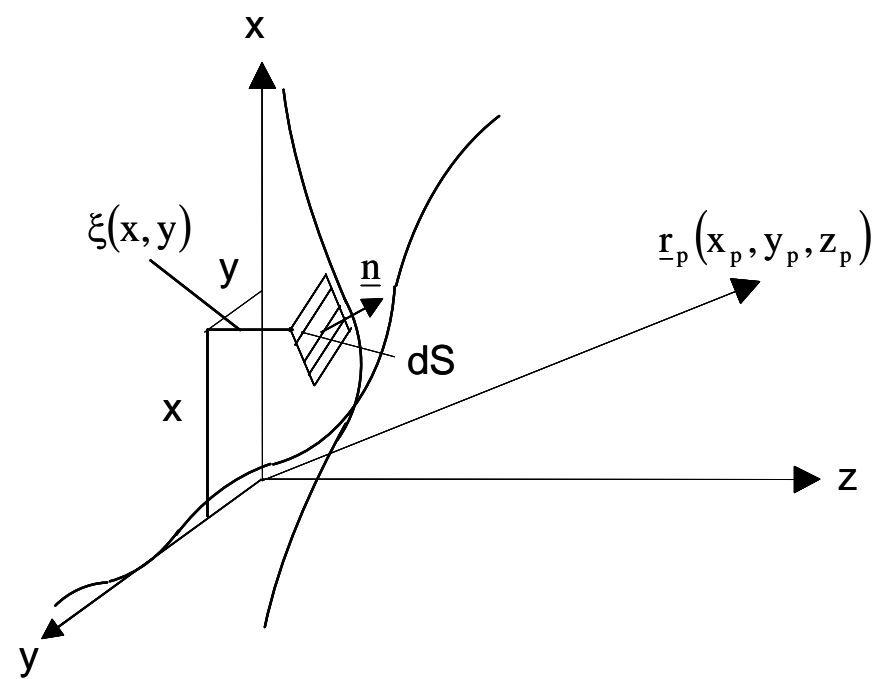

Fig. 1.

after certain algebraic transformation

$$
R=\left(z_{p}-\xi(x, y)\right) \sqrt{1+\left(\frac{x-x_{p}}{z_{p}-\xi}\right)^{2}+\left(\frac{y-y_{p}}{z_{p}-\xi}\right)^{2}}
$$

and

$$
R^{\prime}=\left(z_{p}+\xi(x, y)\right) \sqrt{1+\left(\frac{x-x_{p}}{z_{p}+\xi}\right)^{2}+\left(\frac{y-y_{p}}{z_{p}+\xi}\right)^{2}}
$$

If we take $|\xi|\left\langle\left\langle z_{p}\right.\right.$ into consideration;

$$
\begin{aligned}
& R \cong z_{p} \sqrt{1+\left(\frac{x-x_{p}}{z_{p}}\right)^{2}+\left(\frac{y-y_{p}}{z_{p}}\right)^{2}} \text { and } \\
& R^{\prime} \cong z_{p} \sqrt{1+\left(\frac{x-x_{p}}{z_{p}}\right)^{2}+\left(\frac{y-y_{p}}{z_{p}}\right)^{2}}
\end{aligned}
$$

so in this approximation $R=R^{\prime}$. Replacing these results into the function, we get $g_{p}(x, y, \xi(x, y)) \equiv 0$. Taking this into consideration, the integral expression becomes simpler, namely the second term disappears in the integral formula.

$$
\varphi\left(x_{p}, y_{p}, z_{p}\right)=\frac{1}{4 \pi} \int_{S} \varphi(x, y, \xi(x, y)) \operatorname{grad} g_{p} \mathbf{n} d s
$$

Further simplification is carried out when we give the explicit form of $\operatorname{grad} g_{p}$.

$$
\begin{gathered}
\operatorname{grad} g_{p}=\frac{d}{d R}\left(\frac{e^{i k R}}{R}\right) \frac{\partial R}{\partial \mathbf{r}}-\frac{d}{d R^{\prime}}\left(\frac{e^{i k R^{\prime}}}{R^{\prime}}\right) \frac{\partial R^{\prime}}{\partial \mathbf{r}}= \\
\frac{i k R-1}{R^{2}} \cdot e^{i k R} \frac{\partial R}{\partial \mathbf{r}}-\frac{i k R^{\prime}-1}{R^{\prime 2}} \cdot e^{i k R^{\prime}} \frac{\partial R^{\prime}}{\partial \mathbf{r}}
\end{gathered}
$$

but $\frac{\partial R}{\partial \mathbf{r}}=\frac{\mathbf{r}-\mathbf{r}_{p}}{R} \quad \frac{\partial R^{\prime}}{\partial \mathbf{r}}=\frac{\mathbf{r}-\mathbf{r}_{p-}}{R^{\prime}}$

Since on the surface of $z=\xi(x, y)$, the $R \cong R^{\prime}$, so replacing $\frac{\partial R}{\partial \mathbf{r}}$ and $\frac{\partial R^{\prime}}{\partial \mathbf{r}}$ into the expression of $\left.\operatorname{grad} g_{p}\right|_{S}$ we find $\left.\operatorname{grad} g_{p}\right|_{S}=\frac{e^{i k R^{\prime}}}{R^{\prime}} \frac{i k R^{\prime}-1}{R^{\prime 2}}\left(\mathbf{r}_{p-}-\mathbf{r}_{p}\right)$.

Considering $\left.\left.k R^{\prime}=2 \pi \frac{R^{\prime}}{\lambda}\right\rangle\right\rangle 1$, therefore beside $k R^{\prime}$, the 1 may be left. Writing components of $\mathbf{r}_{p}$ and $\mathbf{r}_{p-}$ in the expression of $\operatorname{grad} g_{p}$, we get:

$$
\left.\operatorname{grad} g_{p}\right|_{S}=\frac{-e^{i k R^{\prime}}}{R^{\prime}} \frac{i k}{R^{\prime}}\left(\begin{array}{l}
0 \\
0 \\
2 z_{p}
\end{array}\right)=\frac{-2 i k}{R^{\prime}} \frac{e^{i k R^{\prime}}}{R^{\prime}}\left(\begin{array}{l}
0 \\
0 \\
z_{p}
\end{array}\right)
$$


Since the $\left.\operatorname{grad} g_{p}\right|_{S}$ has only z component, therefore in the direct product ( $\operatorname{grad} g_{p} \mathbf{n} d S$ ) only the $z$ component of $\mathbf{n} d S$ will give contribution. The $z$ component of $\mathbf{n} d S$ is dxdy, so the integral takes the form:

$$
\varphi\left(x_{p}, y_{p}, z_{p}\right)=-\frac{i}{\lambda} \int_{S} \frac{e^{i k R^{\prime}}}{R^{\prime 2}} z_{p} \varphi(x, y, \xi(x, y)) d x d y
$$

If the illumination happens by a limited beam and $z_{p}$ coordinate of the observation point is much greater than $x, y, x_{p}$ and $y_{p}$, then we can use expansion of $R^{\prime}$

$$
\begin{gathered}
R^{\prime}=\sqrt{\left(x-x_{p}\right)^{2}+\left(y-y_{p}\right)^{2}+\left(\xi(x, y)+z^{p}\right)^{2}}= \\
\left(z_{p}+\xi(x, y)\right) \sqrt{\left(\frac{x-x_{p}}{z_{p}+\xi}\right)^{2}+\left(\frac{y-y_{p}}{z_{p}+\xi}\right)^{2}+1} \cong \\
\cong\left(z_{p}+\xi(x, y)\right)\left\{1+\frac{1}{2}\left[\left(\frac{x-x_{p}}{z_{p}}\right)^{2}+\left(\frac{y-y_{p}}{z_{p}}\right)^{2}\right]\right\}
\end{gathered}
$$

Neglecting the nonlinear terms of $x, y, x_{p}$ and $y_{p}$ in the $R^{\prime}$ we get:

$$
\begin{gathered}
R^{\prime} \cong\left(z_{p}+\xi(x, y)\right)\left(1-\frac{x x_{p}}{z_{p}^{2}}-\frac{y y_{p}}{z_{p}^{2}}\right) \cong \\
z_{p}+\xi(x, y)-\frac{x x_{p}}{z_{p}}-\frac{y y_{p}}{z_{p}}
\end{gathered}
$$

If we replace $\frac{1}{R^{\prime}}$ with $\frac{1}{z_{p}}$ and $e^{i k R^{\prime}}$ with $e^{i k z_{p}} \cdot e^{i k \xi(x, y)}$. $e^{-i k x \frac{x_{p}}{z_{p}}} \cdot e^{-i k y \frac{y_{p}}{z_{p}}}$ then the $\varphi\left(x_{p}, y_{p}, z_{p}\right)$ amplitude takes the following form ;

$$
\begin{gathered}
\varphi\left(x_{p}, y_{p}, z_{p}\right)=-\frac{i}{\lambda} \cdot \frac{e^{i k z_{p}}}{z_{p}} \\
\int_{S} e^{i k \xi(x, y)} \cdot e^{-i k x \frac{x_{p}}{z_{p}}} \cdot e^{-i k y \frac{y_{p}}{z p}} \cdot \varphi(x, y, \xi(x, y)) d x d y
\end{gathered}
$$

Introducing new variables $\frac{x_{p}}{z_{p}}=q$ and $p=\frac{y_{p}}{z_{p}}$ we can get a simpler integral formula:

$$
\begin{gathered}
\varphi\left(p, q, z_{p}\right)=-\frac{i}{\lambda} \cdot \frac{e^{i k z_{p}}}{z_{p}} \int_{S} e^{i k \xi(x, y)} . \\
\cdot e^{-i k q x} \cdot e^{-i k p y} \cdot \varphi(x, y, \xi(x, y)) d x d y
\end{gathered}
$$

This integral formula gives the field-amplitude in the direction determined by the $\mathrm{p}$ and $\mathrm{q}$ around the $\mathrm{z}$ axis, in the $z_{p}$ plane.

The field-amplitude in the integral $\varphi(x, y, \xi(x, y))$ is given by the "d" point of our assumptions.

Consider a plane wave that illuminates the investigated metal surface. Be $\mathbf{e}_{0}$ unit vector parallel with the $y, z$ plane that is directed in the direction of the wave vector of the plane wave. Indicate $\vartheta$ the angle between the $\mathrm{z}$ axis and $\mathbf{e}_{0}$.

According to the Figs $2 \mathrm{a}$, and $2 \mathrm{p}$ the local reflection on the surface is determined by the normal vector and the $\mathbf{e}_{0}$, namely the reflected propagation vector is in the plane of $\mathbf{e}_{0}$ and $\mathbf{n}$. If $\mathbf{e}_{1}$ means the unit vector of the reflected beam, we can claim that the angles between $\left(\begin{array}{ll}\mathbf{e}_{0} & \mathbf{n}\end{array}\right)$ and $\left(\mathbf{e}_{1} \mathbf{n}\right)$ have to be equals. So with the aid of the Fig. 2p we can write the following equations:

$\mathbf{e}_{0}+\mathbf{e}_{1}=\Delta \mathbf{e}$ and $\mathbf{e}_{0}-\mathbf{n}\left(\mathbf{n} \mathbf{e}_{0}\right)=\frac{1}{2} \Delta \mathbf{e}$.

Solving these equations for $\mathbf{e}_{1}$ we get:

$$
\mathbf{e}_{1}=\mathbf{e}_{0}-2 \mathbf{n}\left(\mathbf{n} \mathbf{e}_{0}\right) .
$$

So the reflected beam in an arbitrary point of the metal surface has the following form:

$\varphi(x, y, \xi(x, y))=-A_{0} e^{i k \mathbf{e}_{1} \mathbf{r}}$ where $A_{0}$ is the incoming wave amplitude. The minus sign comes from the $\pi$ phase shift occured at the reflection. This negative sign does not influence the intensity. The surface normal vector is generated by the gradient of the $S=z-\xi(x, y)$ surface, according to the expression $\mathbf{n}=\frac{\operatorname{grad} S}{|\operatorname{grad} S|}$

$$
\operatorname{grad} S=\left(\begin{array}{c}
-\frac{\partial \xi}{\partial x} \\
-\frac{\partial \xi}{\partial y} \\
1
\end{array}\right) \text { so } \mathbf{n}=\frac{1}{\sqrt{\left(\frac{\partial \xi}{\partial x}\right)^{2}+\left(\frac{\partial \xi}{\partial y}\right)^{2}+1}}\left(\begin{array}{c}
-\frac{\partial \xi}{\partial x} \\
-\frac{\partial \xi}{\partial y} \\
1
\end{array}\right)
$$

Substitute $\mathbf{e}_{0}=\left(\begin{array}{c}0 \\ -\sin \vartheta \\ -\cos \vartheta\end{array}\right)$ and $\mathbf{n}$ into the expression of $\mathbf{e}_{1}$

we get: $\mathbf{e}_{1}=\left(\begin{array}{l}0 \\ -\sin \vartheta \\ -\cos \vartheta\end{array}\right)+\frac{2\left(\sin \vartheta \frac{\partial \xi}{\partial y}-\cos \vartheta\right)}{\sqrt{\left(\frac{\partial \xi}{\partial x}\right)^{2}+\left(\frac{\partial \xi}{\partial y}\right)^{2}+1}}\left(\begin{array}{l}\frac{\partial \xi}{\partial x} \\ \frac{\partial \xi}{\partial y} \\ -1\end{array}\right)$

Leaving the first and the second power of $\frac{\partial \xi}{\partial x}$ and $\frac{\partial \xi}{\partial y}$, we find for $\mathbf{e}_{1}=\left(\begin{array}{l}0 \\ -\sin \vartheta \\ \cos \vartheta\end{array}\right)$.

Writing this unit vector into the expression of $\varphi(x, y, \xi(x, y))=-A e^{i k \mathbf{e}_{1} r}$ we get;

$$
\varphi(x, y, \xi(x, y))=-A_{0} e^{i k \mathbf{e}_{1} \mathbf{r}}=-A_{0} e^{i k(-y \sin \vartheta+\xi(x, y) \cos \vartheta)}
$$

Substitute this into the integral formula that gives the following result for the amplitude $\varphi\left(q, p, z_{p}\right)$

$$
\begin{gathered}
\varphi\left(q, p, z_{p}\right)=i \frac{A_{0}}{\lambda} \cdot \frac{e^{i k z_{p}}}{z_{p}} \\
\int_{S} e^{i k \xi(x, y)\{1+\cos \vartheta\}} \cdot e^{-i k y \sin \vartheta} \cdot e^{-i k q x-i k p y} d x d y
\end{gathered}
$$

Since $k \xi(x, y)=2 \pi \frac{\xi(x, y)}{\lambda}$ and at the ultrafine machining $|\xi(x, y)|\left\langle\left\langle\lambda\right.\right.$, therefore the function $e^{i k \xi(x, y)\{1+\cos \vartheta\}}$ can be expanded in Taylor series so $e^{i k \xi(x, y)\{1+\cos \vartheta\}} \cong 1+$ $i k \xi(x, y)\{1+\cos \vartheta\}$. Replacing this into the integral formula leads to:

$$
\begin{gathered}
\varphi\left(q, p, z_{p}\right)=i \frac{A_{0}}{\lambda} \cdot \frac{e^{i k z_{p}}}{z_{p}} \\
\int_{S}[1+i k \xi(x, y)\{1+\cos \vartheta\}] \cdot e^{-i k q x} \cdot e^{-i k(p+\sin \vartheta) y} d x d y
\end{gathered}
$$

So the integral breaks up two terms:

$$
\varphi\left(q, p, z_{p}\right)=i \frac{A_{0}}{\lambda} \frac{e^{i k z_{p}}}{z_{p}} \int_{S} e^{-i k q x} \cdot e^{-i k(p+\sin \vartheta) y} d x d y-
$$




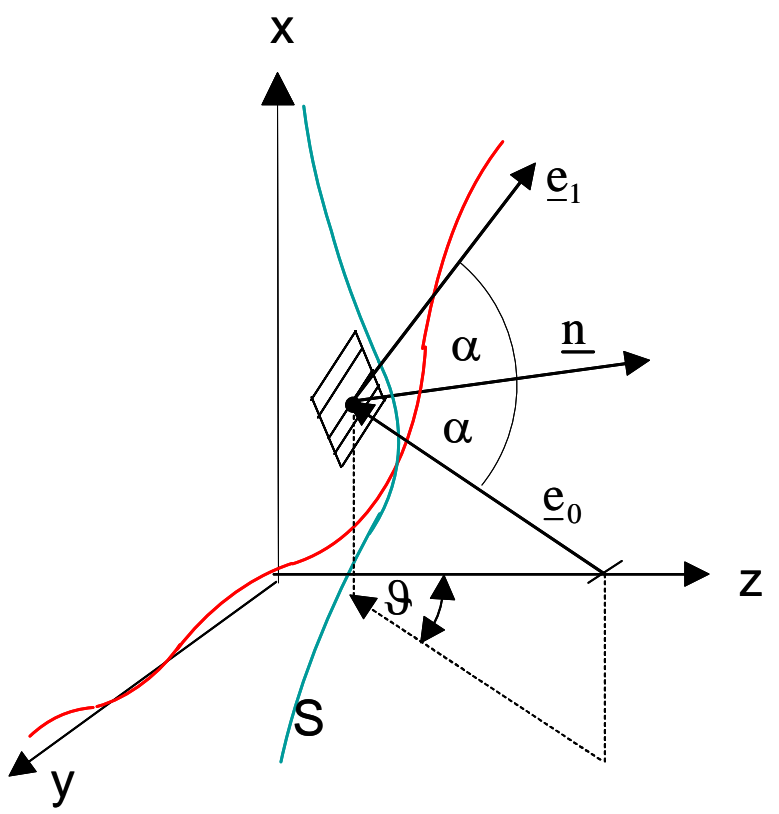

a.

Fig. 2.

$-\frac{A_{0}}{\lambda} \frac{e^{i k z_{p}}}{z_{p}} k(1+\cos \vartheta) \int_{S} \xi(x, y) \cdot e^{-i k q x} \cdot e^{-i k(p+\sin \vartheta) y} d x d y$ The light intensity is given by $\varphi\left(q, p, z_{p}\right) \cdot \varphi^{*}\left(q, p, z_{p}\right)$ namely

$$
\begin{gathered}
\left|\varphi\left(q, p, z_{p}\right)\right|^{2}=\varphi\left(q, p, z_{p}\right) \varphi^{*}\left(q, p, z_{p}\right)= \\
=\frac{A_{0}^{2}}{\lambda^{2}} \frac{1}{z_{p}^{2}} \int_{S} \int_{S} e^{-i k q\left(x-x^{\prime}\right)} \cdot e^{-i k(p+\sin \vartheta)\left(y-y^{\prime}\right)} d x d y d x^{\prime} d y^{\prime}- \\
-i \frac{A_{0}^{2}}{\lambda^{2}} \frac{1}{z_{p}^{2}} k(1+\cos \vartheta) \\
\int_{S} \int_{S} \xi\left(x^{\prime}, y^{\prime}\right) \cdot e^{-i k q\left(x-x^{\prime}\right)} \cdot e^{-i k(p+\sin \vartheta)\left(y-y^{\prime}\right)} d x d y d x^{\prime} d y^{\prime}+ \\
C \cdot C \cdot+\frac{A_{0}^{2}}{\lambda^{2}} \frac{1}{z_{p}^{2}} k^{2}(1+\cos \vartheta)^{2} \cdot \\
\cdot \int_{S} \int_{S} \xi(x, y) \xi\left(x^{\prime}, y^{\prime}\right) \cdot \\
\cdot e^{-i k q\left(x-x^{\prime}\right)} \cdot e^{-i k(p+\sin \vartheta)\left(y-y^{\prime}\right)} d x d y d x^{\prime} d y^{\prime}
\end{gathered}
$$

The sum of the second and third terms is equal to zero, so introducing $p^{\prime}=p+\sin \vartheta$ notation leads to;

$$
\begin{gathered}
\left|\varphi\left(q, p^{\prime}, z_{p}\right)\right|^{2}= \\
=\frac{A_{0}^{2}}{\lambda^{2}} \frac{1}{z_{p}^{2}} \int_{S} \int_{S} e^{-i k q\left(x-x^{\prime}\right)} e^{-i k p^{\prime}\left(y-y^{\prime}\right)} d x d y d x^{\prime} d y^{\prime}+ \\
+\frac{A_{0}^{2}}{\lambda^{2}} \frac{1}{z_{p}^{2}} k^{2}(1+\cos \vartheta)^{2} .
\end{gathered}
$$

$\cdot \int_{S} \int_{S} \xi(x, y) \xi\left(x^{\prime}, y^{\prime}\right) \cdot e^{-i k q\left(x-x^{\prime}\right)} \cdot e^{-i k p^{\prime}\left(y-y^{\prime}\right)} d x d y d x^{\prime} d y^{\prime}$

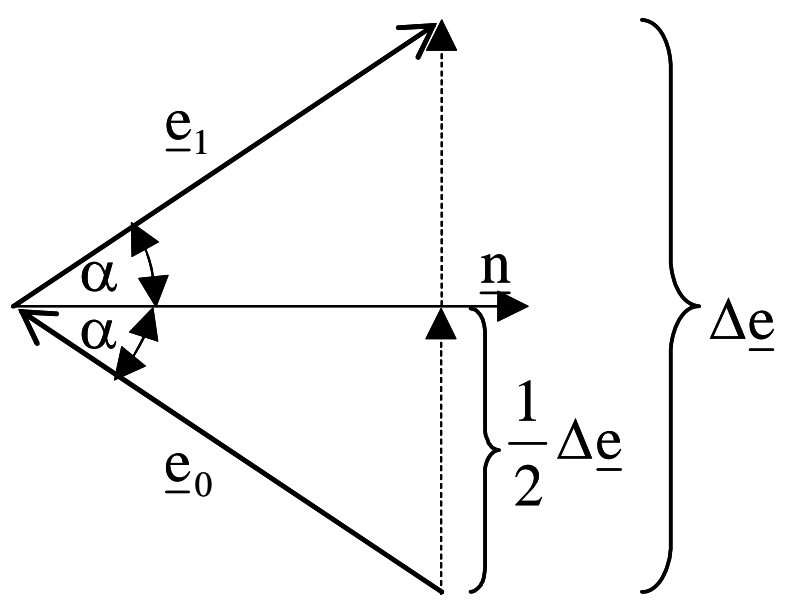

b.

Integrating on all directions $\left(q, p^{\prime}\right)$ then we can make the integration over $x^{\prime}$ and $y^{\prime}$, we obtain the following formula:

$$
\begin{aligned}
& \int\left|\varphi\left(q, p^{\prime} z_{p}\right)\right|^{2} d p^{\prime} d q=\frac{A_{0}^{2}}{\lambda^{2} z_{p}^{2}}\left(\frac{2 \pi}{k}\right)^{2} F+ \\
& +\frac{A_{0}^{2}}{\lambda^{2}} \frac{1}{z_{p}^{2}} k^{2}(1+\cos \vartheta)^{2}\left(\frac{2 \pi}{k}\right)^{2} \int_{S} \xi^{2} d x d y
\end{aligned}
$$

where $F$ is the illuminated metal surface. At the integration over the $p^{\prime}$ and $q$ we utilized the relations;

$$
\begin{gathered}
\int e^{-i k q\left(x-x^{\prime}\right)} d q=\frac{2 \pi}{k} \delta\left(x-x^{\prime}\right) \text { and } \\
\int e^{-i k p^{\prime}\left(x-x^{\prime}\right)} d p^{\prime}=\frac{2 \pi}{k} \delta\left(y-y^{\prime}\right) .
\end{gathered}
$$

In all two terms writing in $\left(\frac{2 \pi}{\lambda}\right)^{2}=k^{2}$ we get;

$$
\begin{gathered}
\int\left|\varphi\left(q, p^{\prime}, z_{p}\right)\right|^{2} d p^{\prime} d q= \\
\frac{A_{0}^{2}}{z_{p}^{2}} F+\frac{A_{0}^{2}}{z_{p}^{2}} k^{2}(1+\cos \vartheta)^{2} \int_{S} \xi^{2}(x, y) d x d y .
\end{gathered}
$$

The first term on the right hand side is proportional to the power of the beam propagating in direction of reflection and the second term is proportional to the power diffracted all different directions.

Introducing the $P_{r}=\alpha \frac{A_{0}^{2}}{z_{p}^{2}} \cdot F$ reflected and $P_{d}=$ $\alpha \frac{A_{0}^{2}}{z_{p}^{2}} k^{2}(1+\cos \vartheta)^{2} \int_{S} \xi^{2}(x, y) d x d y$ diffracted powers where $\alpha$ is an conversation constant, we can write the ratio of $P_{d}$ and $P_{r}$

$$
\frac{P_{d}}{P_{r}}=\frac{k^{2} \sqrt{(1+\cos \vartheta)^{2}}}{F} \int_{S} \xi^{2}(x, y) d x d y .
$$




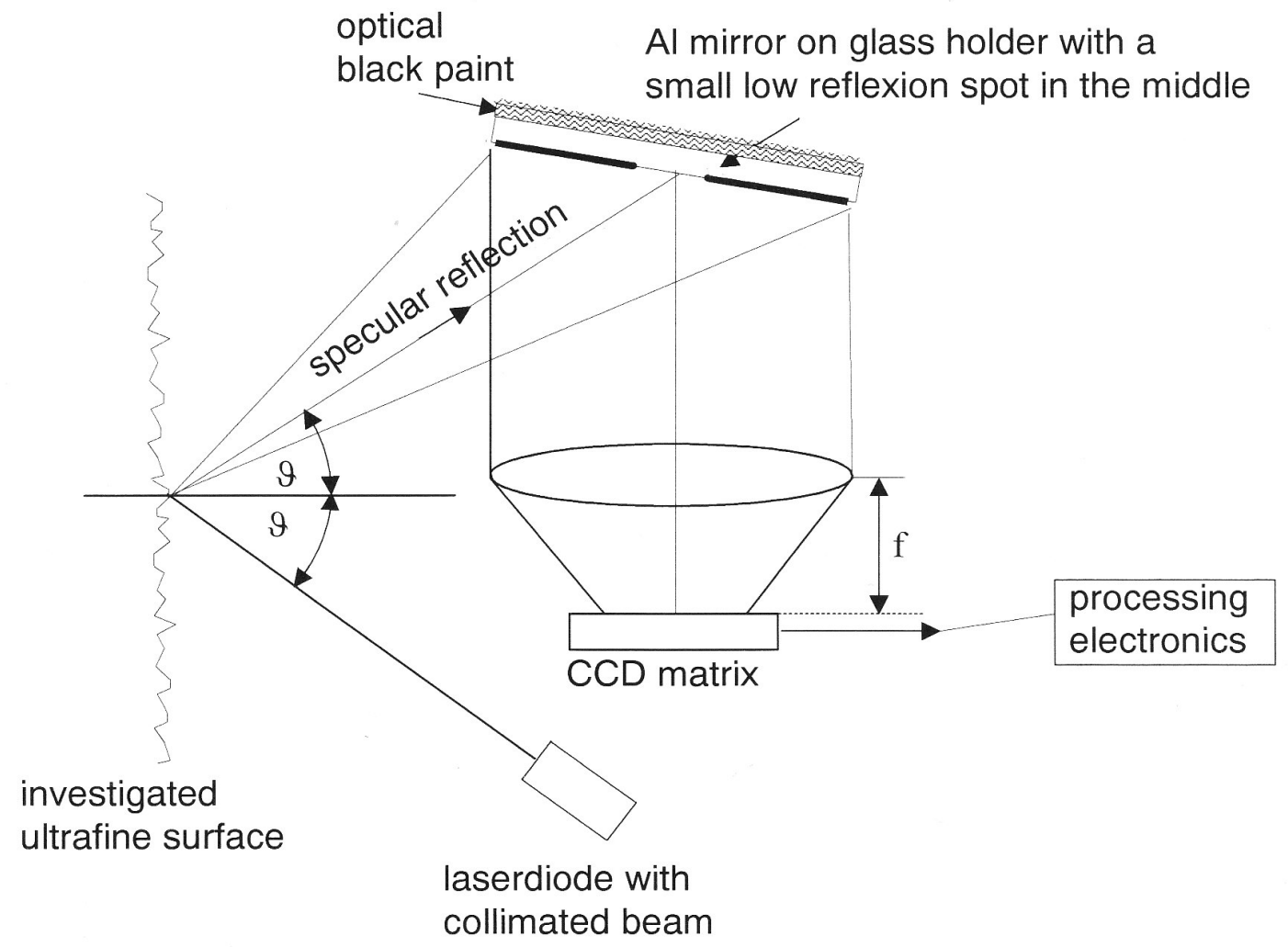

Fig. 3.

If we introduce the $R_{q}=\sqrt{\frac{1}{F} \int_{S} \xi^{2}(x, y) d x d y}$ true average surface roughness, we can rewrite the former equation for $\frac{P_{d}}{P_{r}}$;

$$
\frac{P_{d}}{P_{r}}=k^{2}(1+\cos \vartheta)^{2} R_{a}^{2} .
$$

Expressing the $R_{q}$ from this equation we get:

$$
R_{q}=\frac{\lambda}{2 \pi} \sqrt{\frac{P_{d}}{P_{r}}} \cdot \frac{1}{(1+\cos \vartheta)}
$$

The result shows us that in case of the ultrafine machining, the measurement of the true roughness is reduced to the measurement of the reflected and diffracted powers.

\section{Realization of ultrafine optical roughness measure- ment}

A practicable application version of the method is seen on Fig. 3

The investigated surface is illuminated by a collimated beam of a laser diode under an angle of $\vartheta$. The reflected and diffracted amplitudes are admitted by at least $\frac{\lambda}{20} \mathrm{Al}$ mirror on glass holder with a small low reflection spot in the middle. The small low reflection spot prevents the CCD camera from the saturation, and serves as a low space frequency filter. The lens after this mirror secure the farfield observation on the camera screen, that is placed in the focal plane of the former lens. The optical axis of the lens is co-linear with the reflected beam and intersects the center of the camera screen.

\section{References}

1 Hasunuma H, Nara J, J.Phys. Soc.Japan 11 (1956), 69.

Middleton W E K, Wyszecki G, J. Opt. Soc. Am. 47 (1957), 1020.

Hunter R S, J. Opt. Soc.Am. 36 (1946), 178.

Bennett H E, Porteus J O, J. Opt. Soc. Am. 51 (1961), 123.

H.Davies, Proc.Inst.Elec.Engrs. 101 (1954), 209.

6 Stover J C, Applied Optics 14 (1975), 1796.

7 Jackson J D, Classical Electrodynamics (1975), 430. Sect.9.8. 